\title{
Analyse des difficultés des étudiants jordaniens dans l'écrit scientifique universitaire: le cas du rapport de recherche dans une formation FLE
}

Rana KANDEEL, Université de Yarmouk, Jordanie

L'écrit universitaire en français par des étudiants étrangers a commencé à se développer dans les pays non francophones à l'essor de l'ouverture des disciplines francophones dans les établissements de l'enseignement supérieur. Pour écrire en langue scientifique étrangère il faut avoir un bon niveau linguistique, une appropriation d'une compétence méthodique et une maîtrise d'une compétence disciplinaire dans un domaine spécialisé.

Les travaux didactiques de Jean-Marc Mangiante et Chantal Parpette (2011) sur la méthodologie de l'enseignement du français sur objectif universitaire (FOU) témoignent de l'intérêt de ce champ d'études à l'heure actuelle. En effet, la mobilité croissante des étudiants étrangers dans des pays francophones et le besoin de l'ouverture sur leurs méthodologies d'enseignement ont créé de nouvelles exigences didactiques dans les pays non francophones. Parmi ces exigences, la nécessité d'apprendre aux étudiants dans l'enseignement supérieur les techniques de l'écrit scientifique en français.

Si le FOU est considéré d'un intérêt considérable pour la préparation des étudiants à un futur départ en France, il ne semble pas moins indispensable à la préparation des étudiants étrangers des filières francophones dans leurs pays pour apprendre l'écrit universitaire et le raisonnement à la française, selon Mangiante et Parpette :

Dans les pays où le français est langue de l'enseignement supérieur, la problématique de l'intégration est également pertinente mais doit être nuancée dans la mesure où les étudiants ne sont pas «étrangers » dans leurs universités. Il s'agit d'une forme d'intégration à un environnement de travail et de langue différente de ce qu'ils ont connu auparavant, où la langue étrangère devient langue d'enseignement et de communication universitaire à côté de la langue maternelle ou de la première langue apprise à l'école. (53)

\section{La communication scientifique écrite : un projet à réussir}

La communication scientifique écrite n'est pas une simple transcription des informations recueillies ni une simple présentation des idées. C'est un projet bien élaboré et fondé sur les compétences du chercheur lequel ne doit pas se contenter d'être à la hauteur, il doit montrer ses 
compétences lors de la rédaction du travail scientifique. Les éléments à associer pour la réussite de ce projet sont :

$>$ Savoir (connaissances anciennes----connaissances nouvelles)

$>$ Savoir-faire (méthode d'investigation)

$>$ Faire savoir (communication) (Defays, Maréchal et Saenen 100)

L'interaction entre ces trois éléments joue un rôle crucial dans la production de la communication scientifique. Mais nous nous intéressons ici à la compétence Faire savoir, définie comme la capacité de communiquer les résultats d'une recherche scientifique. Elle est constituée des composantes méthodologiques, des moyens linguistiques et des stratégies discursives puisqu'ils sont «déterminants pour une bonne intégration aux études universitaires dans la mesure où ils préparent les étudiants à la réalité des situations et des discours auxquels ils sont confrontés » (Mangiante et Parpette 27).

\subsection{Le Faire savoir et les compétences méthodologiques}

La rédaction du plan de travail ainsi que l'élaboration du plan de rédaction permettent de mettre en relief les idées principales. La communication scientifique doit inclure des éléments méthodologiques essentiels pour se montrer convaincante. Une démarche intellectuelle de conception, de construction et d'élaboration est à l'origine de la cohérence de ce travail; c'est la structure logique.

La division du travail en introduction, table des matières, parties, chapitres, conclusion qui rend la présentation plus solide et claire est nommée la structure physique. Le choix de la méthode scientifique doit se faire en fonction de la discipline et du sujet. La réflexion sur les potentialités et les limites de la méthode et sur son rôle d'éclairage de la question traitée ne peut jamais se faire sans l'esprit critique du chercheur (Beaud 69).

\subsection{Le Faire savoir et les compétences langagières}

Le langage joue un rôle important dans l'écrit scientifique, ainsi Jean-Marc Defays, Marielle Maréchal et Frédéric Saenen soulignent que : «le langage ne sert pas seulement à traduire le raisonnement de l'auteur mais aussi à le soutenir; qu'il ne vise pas seulement à transmettre des informations au lecteur, mais aussi à proposer de nouvelles perspectives et à créer de nouvelles connaissances » (11). Il faut utiliser une langue bien adaptée aux spécificités techniques ou scientifiques du sujet de la recherche. 
A notre sens, si les exigences méthodologiques s'acquièrent par un étudiant étranger à travers la formation, il n'est pas de même en ce qui concerne le développement de la compétence langagière. La pratique, l'auto-formation et l'évaluation par l'enseignant sont des méthodes efficaces qui conduisent à ce développement.

Un bon choix du vocabulaire permet de présenter des mots riches et précis. Les propriétés de la langue de communication scientifique auxquelles le chercheur doit faire attention sont présentées selon plusieurs aspects (Defays, Maréchal et Saenen 16-32), nous nous contentons d'en évoquer deux:

1- $\quad$ Les termes génériques et les termes spécifiques: L'identification terminologique du champ disciplinaire passe du plus général au plus spécifique par l'utilisation des termes propres au sujet de la recherche (Cislaru, Claudel et Vlad 19). La rigueur conceptuelle implique une bonne maîtrise des notions spécialisées ainsi qu'une capacité de les présenter et de les hiérarchiser.

2- $\quad$ Les ambiguïtés linguistiques: Ce sont des phénomènes qui réduisent la clarté de la présentation du discours ou des idées par l'auteur. Ils relèvent parfois de l'interprétation du texte faite par le lecteur-Ces ambiguïtés linguistiques sont de différents types: lexicales, morphologiques, syntaxiques pragmatiques et complexes. Dans la phrase «Pierre propose à Paul de l'aider », nous constatons une ambiguïté syntaxique car le complément d'objet direct peut renvoyer à tous les deux (Pierre et Paul).

\subsection{Le Faire savoir et les compétences discursives}

Parmi les éléments discursifs dont on doit tenir compte dans l'élaboration de l'écrit scientifique nous nous intéressons au registre de langue et aux maladresses. Le registre de langue permet la distinction entre la langue écrite et la langue orale qui est plus simple et spontanée. Il ne faut pas utiliser un langage familier ni un jargon technique qui rend l'écrit peu lisible. Le chercheur doit préférer une langue soutenue dont l'intérêt peut être distingué au niveau lexical (choix de vocabulaire spécialisé, précis et neutre), syntaxique (respect des normes grammaticales, complexité et variétés des phrases) et discursive (organisation des phrases, argumentation et développement méthodique du travail). Les maladresses sont définies comme un manque d'habilités ou de savoir-faire dans le discours et dans la conduite d'une personne (Lehnen, Dausendschön-Gay et Krafft 123). 
Les stratégies discursives sont également prises en compte dans la démarche de la communication scientifique. Parmi ces stratégies, Defays, Maréchal et Saenen évoquent différents types : le questionnement, la description, la redéfinition, la relation, l'explication, la comparaison, la construction, la création et l'argumentation (106-108). Elles ont des fonctions permettant de développer un travail raisonné et structuré par exemple, le questionnement permet la prise de distance par rapport à son propre travail et aux travaux précédents. Un chercheur qui pose des questions de type : comment, qui, que, pourquoi, quand et où réussit à mieux conceptualiser le sujet étudié.

L'argumentation est une tâche essentielle de la démarche scientifique, elle se développe à l'étape de l'analyse des données. Selon Lawrence Olivier et Jean François Payette: «le chercheur doit débattre avec des énoncés, des affirmations qu'il construit à partir des données qu'il a colligées. Cette structure doit être soutenue par un travail réflexif qui vient expliquer la relation entre les variables ou faire comprendre la signification qui est donnée au phénomène étudié » (4).

\section{Le rapport de recherche : un type d'écrit scientifique}

Notre réflexion vise particulièrement un type de la communication scientifique écrite, à savoir le rapport de recherche.

\section{1. Définition et fonctions du rapport de recherche}

Le rapport de recherche est défini comme « un écrit scientifique plus officiel et plus formel que le compte rendu. Il présuppose un examen plus systématique des différents aspects d'un problème ou d'un sujet, et exige une présentation plus structurée et logique suivant un plan » (Defays, Maréchal et Saenen 96). Il a certaines caractéristiques qui le distinguent d'autres types de l'écrit scientifique. Produit en nombre limité d'exemplaires, le rapport de recherche est rédigé dans le but d'obtenir une rétroaction sur un sujet de la part de spécialistes de la question développée. La rédaction du rapport constitue la dernière étape du processus de la recherche.

Le travail d'évaluation critique rédigé par nos étudiants peut être classé dans la catégorie des rapports de recherche produits pour rendre compte des résultats de recherche à une communauté scientifique (Dubois 12). Au niveau linguistique et des actes langagiers, il peut être placé dans les rubriques «commentaire et synthèse » et «études de cas » dans la typologie des 
productions écrites universitaires faite par Mangiante et Parpette. L'étudiant doit rédiger une synthèse, une analyse d'une situation donnée et une interprétation des données selon des paramètres ponctuels. Les deux catégories nécessitent des outils langagiers spécifiques notamment un lexique spécialisé et «elles font appel à des compétences langagières et discursives globales et transférables aux différentes disciplines » (131).

Les fonctions principales que le rapport de recherche peut assumer sont récapitulées en ce qui suit:

- La fonction d'exposition: elle assure la suite logique de l'ensemble du document.

- La fonction argumentative : qui amène le chercheur à ne pas se contenter de présenter un texte narratif. Le rapport « exerce également une fonction argumentative, en ce sens que l'auteur du rapport doit amener le lecteur à jouer un rôle de «co-analyste » de son processus de recherche et de ses résultats. Il doit le convaincre de la validité de ses conclusions de recherche » (Lessard-Hébert, Goyette et Boutin 86-87).

- $\quad$ La fonction didactique: cette fonction fait du rapport de recherche un processus d'enseignement des résultats dans la mesure où les sujets impliqués dans la recherche pourraient prendre du recul par rapport à leurs pratiques.

\section{2. Les compétences de Faire savoir et les fonctions du rapport de recherche}

Il faut remarquer que les fonctions assurées par le rapport de recherche en 2. 1 . correspondent aux compétences et stratégies de Faire savoir comme le montre le schéma suivant:



Fig. 1 : Les fonctions du rapport de recherche et les compétences de l'écrit scientifique

Les difficultés des étudiants étrangers en France sont définis à trois niveaux : contextuelculturel, linguistique et méthodologique. Le premier niveau concerne les relations des étudiants avec les autres étudiants et avec les professeurs ou l'administration dans un contexte 
universitaire. Le deuxième est le niveau de la langue de spécialité et la langue mise en œuvre dans les différentes situations de communication. Le troisième désigne les aspects méthodologiques dans la réalisation des tâches scientifiques que l'étudiant doit maitriser dans les discours oraux ou écrits (Mangiante et Parpette 52). Etant donné que les étudiants jordaniens suivent une formation dans leur pays d'origine, nous nous intéressons dans le cadre de cette étude aux deux niveaux linguistiques et méthodologiques.

\section{3- La formation FLE comme contexte de la recherche : public et tâche}

Dans le cadre du cours de linguistique appliquée de master sciences du langage, quatre étudiants inscrits dans ce cours pour le deuxième semestre en 2013 ont travaillé sur une proposition de projet pédagogique, une des formes de la mise en œuvre de la perspective actionnelle. Trois étudiants ont une expérience de l'enseignement du FLE dans des établissements jordaniens scolaires et universitaires, le quatrième n'a pas la même expérience professionnelle.

L'enseignement supérieur en Jordanie est assuré essentiellement en arabe, la langue maternelle des étudiants, et dans certains filières scientifiques en anglais, la première langue étrangère en Jordanie. Le français est la langue d'enseignement des filières francophones qui ne sont destinées qu'à l'enseignement de la langue française. Le français est enseigné comme deuxième langue étrangère et elle est une matière facultative dans les écoles publiques. Dans les écoles privées, cette langue est enseignée précocement. Trois étudiants ont étudié le français avant leurs études universitaires.

Les étudiants ont des profils homogènes au niveau des objectifs d'apprentissage et des habitudes antérieures de l'écrit. En général, il s'agit d'un public qui souhaite développer ses compétences linguistiques et professionnelles dans la formation en sciences du langage, spécialité langue française. Bien que les étudiants ne soient pas novices dans l'apprentissage du français car ils ont atteint le niveau B2, ils n'ont pas une riche expérience rédactionnelle puisqu'ils ne pratiquent pas suffisamment l'écrit depuis leurs études universitaires en licence. Les cours d'expression écrite portent plutôt sur l'apprentissage de la grammaire et non sur la rédaction. De plus, la rédaction en langue étrangère au niveau scolaire n'est pas fortement 
présente. L'homogénéité du public rend l'organisation et la proposition des outils d'aide à la pratique de l'écrit plus facile.

Les étudiants sont peu nombreux dans ce cours parce que le programme de master a été créé à l'université du Yarmouk en 2011. Le nombre total des étudiants qui y sont inscrits est de 23. En fait, nous sommes dans l'obligation de conduire notre recherche sur un échantillon réduit, c'est une des limitations de ce travail qui ne saurait être exhaustif, mais seulement représentatif de l'écrit scientifique des étudiants dans un pays où l'enseignement du français et en français est mis en place simultanément aux cours spécialisés dans une filière francophone de l'enseignement supérieur.

Nous avons proposé aux étudiants une tâche de production écrite ayant pour objectif de développer leur esprit critique à travers la pratique de l'écrit universitaire scientifique en français. Il s'agissait de rédiger un rapport de recherche comprenant un travail d'évaluation critique de 2 à 3 pages par chaque étudiant sur un projet proposé par son pair. L'objectif de la rédaction des rapports de recherche dans notre contexte est double. En premier lieu, rendre compte des résultats de recherche des partenaires aux autres étudiants. En second lieu, doter les étudiants-chercheurs des outils afin d'acquérir des compétences générales, des compétences rédactionnelles et des compétences spécifiques en français sur objectifs universitaires telles que : l'ardeur au travail, la curiosité scientifique, l'esprit critique, l'autonomie, la rigueur de raisonnement, le jugement, et l'argumentation.

La rédaction des rapports est considérée comme une micro-tâche qui va mener au bout de leurs parcours académiques à la réalisation d'une macro-tâche; la rédaction d'un mémoire de master.

\section{4- Description du corpus et méthode de l'analyse}

Notre corpus est constitué de 4 rapports critiques de recherches qui ont été collectés dans leur version finale, après leur évaluation faite par nous en tant qu'enseignante de ce cours. Les copies sont notées sur dix points. Nous avons demandé l'autorisation écrite des étudiants afin d'inclure leurs travaux dans notre corpus constitué de huit pages en élargissant l'anonymat des étudiants. Les rapports sont représentés par des lettres et chiffres de E1 à E4 indiquant l'étudiant auteur. 
Les rapports sont rédigés à partir d'une analyse basée sur un outil d'évaluation que nous avons mis à leur disposition pour examiner les écrits de leurs partenaires. Les étudiants ont travaillé sur une grille composée des critères scientifiques inspirés de l'évaluation proposée par Maria Antonia et Bertrand Baschwitz, ils portent sur trois axes essentiels, à savoir le contenu et la méthodologie (pertinence/contenu), la forme (accessibilité/lisibilité) (120-123).

Tableau 1 : grille d'évaluation utilisée par les étudiants

\begin{tabular}{|l|l|}
\hline \multicolumn{2}{|c|}{ Evaluation du contenu et de la méthodologie (pertinence/contenu) } \\
\hline Problématique & $\begin{array}{l}\text {-Le travail aborde le thème de la recherche et peut nourrir ma réflexion } \\
\text {-L'introduction permet de cerner l'objet de l'étude, la problématique, sa } \\
\text { pertinence face aux objectifs déclarés } \\
\text {-Il y a une partie au début de travail consacrée à l'état de la question } \\
\text { dans les recherches actuelles } \\
\text {-Le contenu du travail correspond au titre } \\
\text {-Les principales problématiques du sujet sont traitées } \\
\text { - Les questions théoriques sont bien exposées, claires } \\
\text {-Le développement ne présente pas de hors sujet } \\
\text {-Les notions et concepts utilisés sont définis }\end{array}$ \\
\hline Argumentation & $\begin{array}{l}\text {-Elle est présente } \\
\text {-Elle est claire; l'auteur ne mélange pas des arguments moraux et des } \\
\text { arguments scientifiques }\end{array}$ \\
\hline Méthodologie & $\begin{array}{l}\text {-La méthodologie retenue est présentée } \\
\text {-La méthodologie utilisée est adéquate au sujet étudié } \\
\text {-La méthode de collecte et de traitement des données est présentée } \\
\text {-Les données ont une certaine homogénéité } \\
\text {-Les analyses sont bien menées } \\
\text {-Les résultats sont bien présentés ; recours à des moyens graphiques }\end{array}$ \\
\hline Evaluation de la forme (accessibilité/lisibilité) \\
\hline Présentation & $\begin{array}{l}\text {-Le travail possède une introduction et une conclusion } \\
\text {-Le plan du travail est annoncé } \\
\text {-Les titres reflètent le contenu des parties et des chapitres } \\
\text {-Les transitions sont soignées ou inexistantes } \\
\text {-La bibliographie est fournie et bien organisée } \\
\text { - Les annexes sont bien présentées, numérotées, portent un titre clair }\end{array}$ \\
\hline
\end{tabular}

Pour analyser notre corpus, nous avons utilisé une grille permettant de traiter les compétences langagières et méthodologiques susceptibles d'être mises en œuvre dans la rédaction de l'écrit scientifique à partir de différents travaux menés sur ce sujet, inclus dans notre 
cadre théorique. Ce choix est motivé par la volonté de repérer les points faibles de l'écrit du rapport de recherche afin de déterminer les outils d'aide à la production des textes répondant aux attentes. L'accent est mis sur les compétences suivantes :

- $\quad$ méthodologiques: la structure physique et la structure logique.

- langagières : le choix des mots (les termes génériques et les termes spécifiques, les ambiguïtés linguistiques) et les structures langagières.

- discursives: les éléments discursifs (le registre de langue, les maladresses) et l'emploi de différents stratégies telles que le questionnement, l'argumentation, etc.

\section{5- Résultats de la recherche}

Les résultats montrent les difficultés auxquelles font face les étudiants dans leur rédaction. Nous les illustrons à travers des extraits représentatifs des textes des étudiants en gardant les erreurs commises dans leurs travaux originaux.

\section{1. Les caractéristiques communes des aspects méthodologiques des textes}

Les textes sont évalués selon deux types de structure : la structure physique et la structure logique. La première est composée de l'introduction, du développement et de la conclusion. La deuxième est constituée des démarches intellectuelles de conception, de construction et d'élaboration qui sont à l'origine de la cohérence de travail. Les rapports de tous les étudiants commencent par une partie introductive en présentant le sujet du travail qui est l'évaluation des projets de partenaires sans évoquer des savoirs antérieurs sur le sujet. E. 3. « Je vais faire une évaluation pour le projet de X. Ce projet est intitulé sous le vêtement pour différentes conditions météorologiques».

Dans cette partie, une seule étudiante a présenté un rappel des connaissances sur l'intérêt de l'évaluation en tant qu'outil permettant l'amélioration constante des actions ce qui rend son texte clair à travers la précision de l'objectif de son rapport de recherche. Trois étudiants ne font pas l'annonce du plan ou de la méthodologie adoptée dans leurs travaux. Une seule étudiante s'est attachée à exposer brièvement le plan de son travail : E. 2. "Mon évaluation est divisée en 3 parties qui sont: l'évaluation de la forme, l'évaluation de la méthode et l'évaluation du contenu». 
La conclusion était absente des travaux des étudiants. Ceux-ci sont incapables de structurer physiquement le texte. La partie développement était sans organisation chez certains d'entre eux. Ils n'indiquent pas le titre des sujets traités et leur texte n'a pas de sous-titres, ce qui témoigne de leur incapacité à découper ce dernier en paragraphes. La majorité d'entre eux ont écrit un texte en bloc, c'est-à-dire formé d'une seule unité. Malgré la défaillance de la structure physique, nous remarquons que la démarche de conception des rapports, de construction et d'élaboration sous-entend les trois axes essentiels présentés dans la grille d'analyse (forme, méthodologie et contenu) qu'on leur avait distribuée. Les étudiants énumèrent ces aspects dans la structure logique du texte scientifique.

Au niveau du contenu, certains ont critiqué l'ambiguïté de la présentation des idées E. 2. «D’abord, je commence par la forme. La présentation générale du travail n'est pas satisfaisante. La page de couverture n'est pas bien organisée. », ainsi que le choix des mots spécialisés E. 3. «Leçon un il me semble que c'est un cours du français très traditionnel, après moi je ne comprends pas ça veut dire quoi compétence à souligner, structure cible, vocabulaire cible ». D'autres étudiants ont fait une analyse linguistique et ils se sont concentrés sur les fautes d'orthographe et de conjugaison : E. 4. «page 2, dans la partie (une nouvelle démarche), nous remarquons beaucoup de fautes de conjugaison et d'orthographe ». L'absence des projets dans les travaux de leurs partenaires a été aussi critiquée E. 4. «Les éléments tels que (le niveau, contexte, présentation des participants, langue de communication) tout cela m'apparait clair, sauf le projet lui-même, où est le projet? ».

En général, les parties de leur travail critique se concentrant sur la forme semblent prédominantes. Les parties d'analyse du contenu et de la méthodologie sont très courtes. L'absence d'annonce du plan de la rédaction et de la méthodologie adoptée était frappante dans les rapports.

\section{2. Analyse langagière de l'écrit scientifique}

Pour analyser les textes langagièrement, nous avons choisi de travailler sur plusieurs entrées : la terminologie disciplinaire, les ambiguïtés linguistiques et les structures langagières.

\section{- La terminologie disciplinaire}

Les étudiants ont employé les termes génériques comme «projet», «perspective actionnelle » et les termes spécifiques tels que «tâches », «action », « savoir », « savoir-faire » 
qui représentent l'objet de leur critique de façon appropriée. En général, les relations entre les deux types de termes mis en œuvre par les étudiants sont hiérarchiques et associatives.

La relation hiérarchique (parent-enfant) désigne que les termes spécifiques (enfant) précisent la portée des termes génériques (parent) comme dans l'extrait de l'étudiant E.2. «Où est l'action dans le projet? L'objectif essentiel de ce projet qui doit être basé sur la perspective actionnelle est l'action. Alors, ce projet n'a pas d'objectif ». On peut y avoir une hiérarchie entre «la perspective actionnelle» et «l'action». La relation associative propose le renvoi vers d'autre vocabulaire permettant de travailler sur un sujet en rapport. Les mots «savoir» et «savoir-faire» sont associés à la notion «projet»: E. 1. «X a proposé un projet devra appliquer une nouvelle stratégie d'apprendre une langue étrangère (langue française). Une démarche réalisée par la perspective actionnelle qui vise l'acquisition des nouvelles connaissances, de savoir et savoir faire à travers une activité authentique et réelle loin de manuel traditionnel ».

\section{- Les ambiguïtés linguistiques}

C'est un phénomène qui se répète dans l'écrit des étudiants à plusieurs niveaux. Au niveau lexical, nous remarquons l'utilisation des mots ou des phrases pour présenter un jugement sans bien se référer au sujet sur lequel ils le portent. Dans la phrase de E. 3. «Page 4 nous remarquons qu'elle a mis le mot échauffement en minuscule aussi pas très claire qu'est-ce que cela signifie», nous constatons une ambiguïté lexicale car la phrase «qu'est-ce que cela signifie » peut renvoyer à l'explication du sens du mot «échauffement» ou à la mise en minuscule de ce mot, considérée comme faute d'orthographe.

Au niveau syntaxique, certains étudiants utilisent des pronoms personnels ou des adjectifs possessifs sans avoir un référent dans le texte comme c'est le cas dans les phrases de l'étudiant E. 1. «Le travail, je le vois un peu satisfaisante, je ne trouve pas la table de matière pour me déplacer vers la page ou le sujet cherché ou au moins pour savoir le contenu de sa recherche. À l'intérieur du contenu, Il a donné une bonne explication de la perspective actionnelle mais sans baser sur une référence linguistique qui parle de cette approche».

\section{- $\quad$ Les structures langagières}

Les structures langagières employées pour représenter les règles de l'écrit scientifique ne semblent pas suffisamment acquises par la majorité des étudiants qui a pourtant suivi au moins 
deux semestres d'études de master avant de rédiger ce travail. L'étudiant E. 1. écrit « Le projet prend le titre...» pour dire «le titre du projet est...» ou «le projet est intitulé... ». Nous remarquons aussi que cette structure est influencée par la structure la langue maternelle des étudiants, la langue arabe, qui autorise cet emploi au niveau sémantique pour désigner le titre d'un ouvrage ou d'un article.

Le non emploi de mots spécialisés à l'écrit scientifique est remarqué aussi, par exemple le mot « parler » est répétée pour designer « aborder » ou « porter sur » dans les phrases de certains étudiants E. 3. «Il a donné une bonne explication de la perspective actionnelle mais sans baser sur une référence linguistique qui parle de cette approche ».

La répétition des mêmes mots dans une même partie ou paragraphe affaiblit la structure langagière du travail comme le montre l'extrait d'un étudiant qui répète les mots «projet » et « évaluation, évaluer» dans la partie introductive de son rapport E. 4. «Je vais évaluer le projet d'une étudiante....pour commencer mon évaluation, je vais évaluer la forme de ce projet. Le projet a une bonne présentation.... ». La difficulté de l'utilisation des synonymes peut être expliquée non seulement par l'insuffisance du bagage langagier des étudiants jordaniens mais aussi par le manque de pratique de l'écrit scientifique en français ainsi que par le manque de la formation à ce type d'écrit.

Les fautes d'orthographe et les erreurs syntaxiques apparaissent dans des extraits tels que E. 3. «La page a numeroté aussi » (absence de l'accent), E. 1. « il n'a pas déterminé le temps dont le projet a besoin. Parce que réaliser un tel projet prend beaucoup de temps » (utilisation inappropriée des signes de ponctuation), E. 4. « en ce qui concerne le projet, beaucoup de fautes d'orthographe et de phrases incompréhensibles» (pas de respect des règles grammaticales, phrase sans verbe). Par ailleurs, les étudiants sont incapables de construire des phrases syntaxiquement correctes, voire achevées E. 1. «concernant la mise en page est maladroite ».

\section{3. Les pratiques discursives}

Une compétence discursive réside dans la capacité de l'utilisateur/apprenant d'ordonner les phrases en séquences afin de produire des ensembles cohérents (Conseil de l'Europe 96). Elle recouvre l'aptitude à gérer et à structurer le discours en termes de style et de registre, la prise en compte de la qualité de discours. 
En tenant compte de cette définition, le dysfonctionnement observable concerne le registre de langue et, tout particulièrement, l'utilisation d'une langue familière, orale et spontanée. Le mot « registre » renvoie aux différences systématiques entre les variétés de langues utilisées dans des contextes différents (Conseil de l'Europe 94). Nous nous attacherons ici aux niveaux de formulation (officiel, formel, familier, neutre, etc). Les manières d'interrogation interindividuelle posent les problèmes de l'emploi de l'oral écrit. Les étudiants écrivent ce qu'ils pensent ou veulent dire à leurs partenaires, ils ont tendance à croire qu'écrire consiste simplement à mettre par écrit ce que l'on sait dire à l'oral sans adapter le niveau d'expression formelle aux circonstances.

De plus, ils ne semblent pas conscients de la rupture entre la façon dont on communique à l'oral et la façon dont on communique à l'écrit. Cette rupture n'est pas seulement technique, mais elle concerne les types de communication et les usages fondamentaux de la langue écrite par rapport à ceux de la langue orale. Les exemples illustrent bien cette difficulté E. 1. «Autre chose dans la même page elle est mal utilisé le mot article de vêtement ça veut dire quoi ça?», E. 4. «Page 3, (on récapitule ce point de vue sur la perspective actionnelle [....] en accomplissant une "tâche" communicative qu'elle lui mener à bien en développant de apptitides capable à comprendre et à produire un autre). Un autre quoi ? ». Ce type d'écrit n'est ni pensé, ni réorganisé, il semble ne pas avoir été lu et relu par son auteur avant d'être communiqué. Il est considéré comme spontané, tracé à la va-vite, sur le vif (Doquet-Lacoste 11). Il conserve des formes orales et paraît relever, du point de vue des éléments syntaxiques et lexicaux, d'un mélange de langue orale et de langue écrite.

L'utilisation de l'oral dans une communication écrite est due d'une part à la méconnaissance du changement des modalités générales de la communication quand on passe à l'écrit en langue étrangère et elle est liée d'autre part, aux questions de nouveauté et de nonfamiliarité avec des registres plus formels. La production écrite adéquate est le résultat de fréquentation assidue des textes qui permet à l'étudiant d'acquérir un discours écrit et d'éviter de penser son texte dans sa langue maternelle et de le traduire comme il le peut dans la langue cible (Courtillon 74).

Les maladresses sont représentées par l'utilisation des mots vagues E. 1.« Les notions et les concepts difficiles ne sont pas trouvés» et des phrases abstraites E.3. «Page 5, elle écrit 20 
minutes après ça il y a une question, je n'ai pas compris pour quoi mettre la question à la nouvelle ligne? La question est confuse, après la caisse le paragraphe est commencé sans mettre une espace». Les formules de politesse sont utilisées dans le rapport comme dans la partie conclusive de l'étudiant E. 4. «Pour conclure, je vais insérer une copie du travail avec les fautes marquer en rouge et une correction écrite à la main. Merci ».

Concernant le choix des stratégies discursives œuvrées dans l'écrit, il importe de dégager les tendances rédactionnelles indépendantes des traits stylistiques individuels. Les étudiants ont fait une description claire ainsi qu'un développement thématique de leurs sujets, E. 1. «Le travail n'a aucune introduction ni un petit résumé... ». L'argumentation n'apparaît que très peu en comparaison avec la description. E. 2. «Nous évitons dans le travail universitaire le pronoms “on" parce que cela créé une confusion chez le lecteur sur le locuteur auquel on se réfère. C'est préférable de le remplacer par "nous"». La phrase de l'étudiant E. 4. témoigne de son incapacité à argumenter son discours quand il n'utilise pas les marqueurs logiques et il recourt à la stratégie du questionnement au lieu de justifier ses propos par l'utilisation de "parce que »: «je trouve le paragraphe précédent mal dit, ex: (devra l'accomplir) qui ? macro tâche ou micro tâche». Le questionnement semble prédominante et il est mis en œuvre pour remplacer d'autres stratégies comme nous venons de l'évoquer.

\section{En guise de conclusion}

A l'issue des résultats de l'analyse, nous constatons que les étudiants ont plus de difficultés dans la présentation de la structure physique des rapports que dans la présentation de leur structure logique. En tenant compte des compétences langagières, le dysfonctionnement observable concerne l'insuffisance des niveaux linguistiques (non-respect des normes grammaticales, défaut de la structure syntaxique des phrases, absence de la ponctuation, etc.).

Les ambiguités linguistiques lexicales et syntaxiques ainsi que l'insuffisance de l'acquisition des structures langagières employées pour représenter les règles de l'écrit scientifique constituent les autres aspects des difficultés langagières. Enfin, l'absence d'une langue soutenue, tout particulièrement, l'utilisation d'une langue orale et spontanée, les maladresses et la concentration sur la description plus que sur l'argumentation semblent principalement les défauts de l'écrit du point de vue discursif. 
Bien que ces résultats soient préliminaires, ils offrent certaines pistes réflexives. En effet, le manque de pratique de l'écrit en langue maternelle des étudiants ainsi qu'en langue étrangère dans le système éducatif scolaire et celui de l'enseignement supérieur en Jordanie rend plus compliqué le processus de développement des compétences méthodologiques, langagières et discursives. Ce fait réduit la possibilité d'adaptabilité des discours des étudiants aux écrits scientifiques et fait émerger le besoin d'une formation spécifique aux genres de l'écrit universitaire.

Dans la rédaction de rapport de recherche, la relation entre la pratique de l'écrit et la réflexion sur l'écrit n'a pas formulé une démarche réflexive chez les étudiants qui sont euxmêmes enseignants FLE. Bien que les travaux des partenaires se soient concentrés sur la forme, la réflexion sur cet élément de leur propre écrit scientifique n'est malheureusement pas apparue dans la pratique de la majorité quand ils ont fait la rédaction critique. Autrement dit, il est intéressant de remarquer que tout en étant incapables de faire mieux, les étudiants n'ont pas eu conscience que leur production était inadéquate. L'approche que nous avons mise en œuvre sousentendait la formation et l'autoformation à l'écrit scientifique par l'examen des travaux des autres étudiants. Mais, pour un public qui n'a pas déjà pratiqué l'écrit scientifique ou ni n'en a eu l'habitude, le processus de travail devient complexe et difficile. La version d'autonomie d'expertise à l'écrit est donc rejetée telle que soulignée par Christiane Donahue (137-160).

Les travaux de recherches des étudiants et les démarches qui y sont adoptées ne peuvent pas seulement être considérés comme le résultat d'un travail personnel. La pratique des enseignants dans ses formes méthodologique, organisationnelle et culturelle influe sur la communication scientifique pratiquée à l'université, principalement à l'écrit. Le développement des compétences des étudiants dépend de la formation des enseignants. Les étudiants ne semblent pas être capables de s'orienter vers un modèle de texte scientifique, en l'occurrence le rapport de recherche, puisqu'ils n'ont pas probablement développé ce modèle en lisant, en analysant et en rédigeant des textes spécialisés. Cette idée incite à adopter une approche d'accompagnement des étudiants dans la réalisation des activités ayant pour objectif l'analyse explicite des communications scientifiques puisque notre public n'est pas habitué à la lecture dans ses études universitaires ou scolaires. L'objectif de cette démarche est de développer des connaissances sur les normes de la production scientifique. 
Le questionnement est une stratégie indiquant le trait de l'oral dans le texte écrit mais aussi il est utilisé pour remplacer les stratégies argumentatives. C'est la raison pour laquelle la fonction argumentative de la recherche n'a pas paru dans les textes. Il est recommandé donc de former à l'argumentation.

\section{Bibliographie}

Antonia, Maria et Bertrand Baschwitz. Comment me documenter ? Bruxelles : De Boeck, 2010.

Beaud, Michel. L'art de la thèse, comment préparer et rédiger un mémoire de master, une thèse de doctorat ou tout autre travail universitaire à l'ère du Net. Paris : La découverte, 1985.

Cislaru, Georgeta, Chantal Claudel et Monica Vlad. L'écrit universitaire en pratique. Bruxelles : De Boeck, 2009.

Conseil de l'Europe. Un cadre européen commun de référence pour les langues : apprendre, enseigner, évaluer. Strasbourg : Division des politiques linguistiques, 2000.

Courtillon, Jeanine. Elaborer un cours de fle. Paris : Hachette, 2003.

Defays, Jean-Marc, Marielle Maréchal et Frédéric Saenen. Principes et pratiques de la communication scientifique et technique. Bruxelles : De Boeck, 2003.

Donahue, Christiane. «Évolution des pratiques et des discours sur l'écrit à l'université : étude de cas ». Lidil 41 (2010) : 137-160.

Doquet-Lacoste, Claire. «Écrits intermédiaires, écritures intermittentes. Carnets, notes, bribes de science ». Langage et société 1. 127 (2009) : 7-22.

Dubois, Jean-Marie M. La rédaction scientifique, Mémoires et thèses : formes régulière et par articles. Issy- les-Moulineaux : ESTEM, 2005.

Lehnen, Katrin, Ulrich Dausendschön-Gay et Ulrich Krafft. « Comment concevoir l'acquisition d'une compétence rédactionnelle pour des textes de spécialité ? " Acquisition et interaction en langue étrangère 12 (2000) : 123-145.

Lessard-Hébert, Michelle, Gabrielle Goyette et Gérald Boutin. La recherche qualitative: fondements et pratiques. Montréal : AMS, 1997.

Mangiante, Jean-Marc et Chantal Parpette. Le français sur objectif universitaire. Grenoble : PU de Grenoble, 2011.

Olivier, Lawrence et Jean François Payette. Argumenter son mémoire ou sa thèse. Canada : PU du Québec, 2010. 